\title{
Delayed Pneumothorax After Bronchoscopy in a Lung Transplant Patient
}

\author{
Alan C Kwan, Sudhakar Pipavath MD, Peter J Leary MD, and Gregory Kicska MD PhD
}

\begin{abstract}
Lung transplant patients commonly undergo transbronchial biopsy to evaluate for rejection. Postbiopsy radiographs are used to exclude pneumothorax, one of the most common major complications. We report a lung transplant patient who developed a pneumothorax 5 months after transbronchial biopsy, with multiple intervening chest computed tomograms documenting that the pneumothorax developed from the biopsy site. This case illustrates that in transplant patients transbronchial biopsy can evolve to pneumothorax several months later, despite normal post-biopsy radiographs. Key words: lung transplant; transbronchial biopsy; transplant rejection; pneumothorax. [Respir Care 2013;58(3):e18-e19. (C) 2013 Daedalus Enterprises]
\end{abstract}

\section{Introduction}

Lung transplant patients commonly undergo transbronchial biopsy to evaluate for rejection. Post-biopsy radiographs are used to exclude pneumothorax, one of the most common major complications. We report a lung transplant patient who developed a pneumothorax 5 months after transbronchial biopsy. Multiple intervening chest computed tomograms (CTs) document that the pneumothorax developed from the biopsy site. This case illustrates that in transplant patients, transbronchial biopsy can lead to an atypical evolution of pneumothorax, delaying classical radiographic and clinical manifestations for several months.

\section{Case Report}

A 26-year-old woman with cystic fibrosis presented with classical findings and symptoms of pneumothorax 5 months after a transbronchial biopsy and 18 months following bilateral lung transplant.

Mr Kwan is affiliated with the School of Medicine; Drs Pipavath and Kicska are affiliated with the Department of Radiology; and Dr Leary is affiliated with the Department of Pulmonary and Critical Care Medicine, University of Washington, Seattle, Washington.

Dr Pipavath has disclosed a relationship with GE Healthcare and Amrsys Publishers. The other authors have disclosed no conflicts of interest.

Correspondence: Gregory Kicska MD PhD, Department of Radiology, University of Washington Medical Center, 1959 NE Pacific Street, Box 357115, Seattle WA 98195-7115. E-mail: kicskag@uw.edu.

DOI: $10.4187 /$ respcare. 01842
The patient's post-transplant course was complicated by cryptogenic organizing pneumonia, multiple episodes of acute rejection, and a sternal fluid collection. The patient was placed on $10 \mathrm{mg}$ prednisone daily. Thirteen months following transplant the patient presented with bilateral pulmonary infiltrates and hypoxia. Right lower lobe transbronchial biopsy was performed, demonstrating organizing pneumonia, without evidence of rejection. CT performed 4 weeks later showed a new $5 \mathrm{~mm}$ cyst at the biopsy site (Figure). The cyst margins were approximately $1 \mathrm{~cm}$ from the pleura and surrounded by normal lung parenchyma. Subsequent CT exams at 1,2, and 4 months that followed up a sternal fluid collection incidentally showed progressive enlargement of the cyst and eventual extension of air to the pleural surface. Chest radiographs during this period were normal.

Five months after the biopsy the patient presented with sudden onset shortness of breath and hypoxemia. Chest radiograph showed a right-sided pneumothorax. CT showed enlargement of the right lower lobe cyst in communication with new visceral sub-pleural blebs. A break in the visceral pleura was identified and was thought to represent the source of the pneumothorax.

The patient ultimately had 3 recurrent right-sided pneumothoraces requiring thoracostomy tube evacuation of the air. Finally, prednisone was tapered to facilitate healing, and the patient has been pneumothorax free for 6 months.

\section{Discussion}

Transbronchial lung biopsy is common in the lung transplant population, in order to detect acute rejection. ${ }^{1}$ The 


\section{Delayed Pneumothorax After Bronchoscopy in a Lung Transplant Patient}

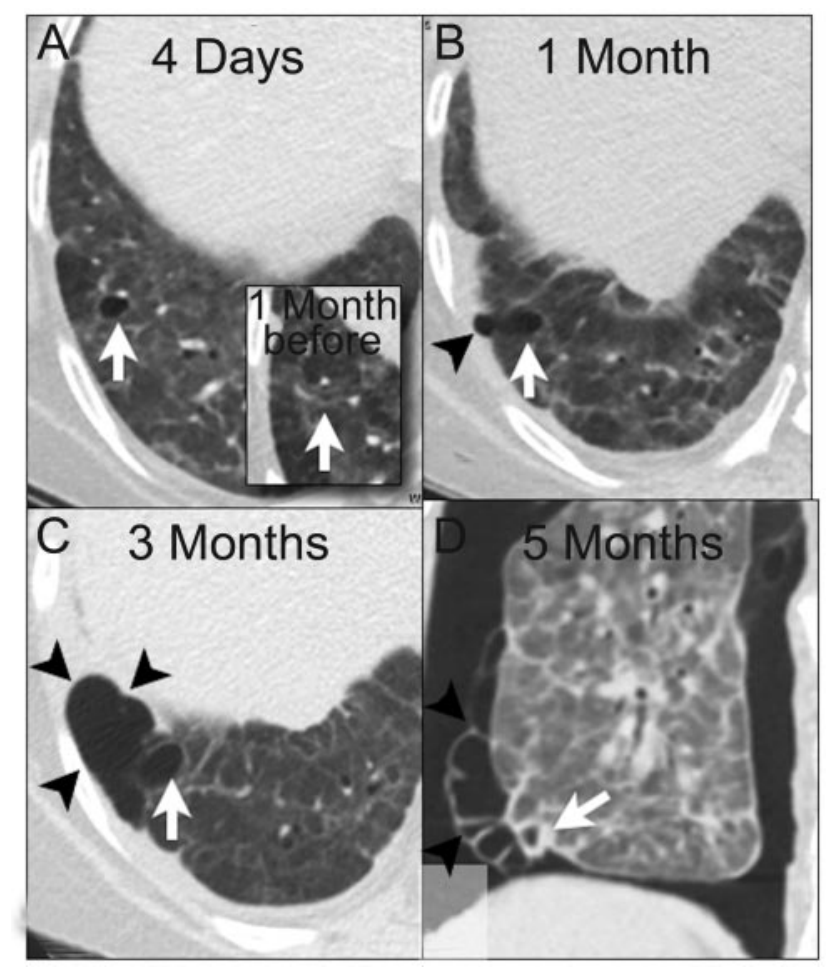

Figure. A: Initial unenhanced chest computed tomogram (CT), performed 4 days after transbronchial lung biopsy. Thin walled cavity/ lucency/pneumatocele demonstrates a new defect that was not present on prior studies and represented the biopsy site. Image (inset) 1 month before biopsy showed no defect. B: Unenhanced CT of the chest done 1 month after biopsy shows mild expansion of the cavity that extends into a collection of air (white arrow), encysted within the sub-pleural lung. C: CT 3 months post-biopsy shows continued expansion of original biopsy site (arrowheads), with marked increase in sub-pleural collection of air (white arrow). D: Unenhanced CT of the chest, coronal reformat, done 5 months after biopsy, shows original biopsy defect (arrowheads), local dehiscence of the visceral pleura, blebs (arrow), and a large pneumothorax.

risk of acute pneumothorax in lung transplant patients after transbronchial biopsy is estimated to be $0-1.5 \%,{ }^{2}$ making it a relatively safe and effective diagnostic tool. Delayed pneumothorax is extremely rare and can occur from a number of different procedures, either directly or inadvertently involving the lung. ${ }^{3-5}$ While delayed pneumothorax has been previously reported, our report is unique due to the magnitude of elapsed time between the transbronchial biopsy and the development of the pneumothorax, and the clear evolution documented on imaging.

Past reports have focused primarily on displacement or fibrinolysis of blood clots as the most likely mechanism of delayed pneumothorax. Other reported associations include infection or emphysema. ${ }^{3-5}$ However, this case represents a different mechanism: the slow evolution of damaged, friable lung tissue to an at risk cystic area of lung parenchyma.

Enlargement of the original defect at the biopsy site could be due to a variety of factors, including an ongoing air leak, infection, immune reaction, or medications. ${ }^{6} \mathrm{Mi}-$ crobes may have seeded the biopsy defect, increasing the probability of pneumothorax. Likewise, cryptogenic organizing pneumonitis and prednisone may also have played a role, as both are associated with poor wound healing and parenchymal frailty pneumothoraces. ${ }^{7-9}$ The biopsy may have caused a flap of tissue that obstructed air flow during exhalation, which persisted due to poor healing. Pertinent negatives include no radiographic or laboratory evidence of Pneumocystis jirovecii by immunofluorescence assay stain. The patient denied any history of smoking or illicit drug use.

This case study illustrates the complexity of lung transplant patients. It demonstrates a uniquely visualized progression of damage to the lung over time, culminating in a prolonged hospitalization for pneumothorax 5 months after the initial procedure.

\section{REFERENCES}

1. Arcasoy SM, Kotloff RM. Lung transplantation. N Engl J Med 1999; 340(14):1081-1091.

2. McWilliams TJ, Williams TJ, Whitford HM, Snell GI. Surveillance bronchoscopy in lung transplant recipients: risk versus benefit. J Heart Lung Transplant 2008;27(11):1203-1209.

3. Levy H, Kallenbach JM, Feldman C, Pincus P, Hurwitz M. Delayed pneumothorax after transbronchial lung biopsy. Thorax 1986;41(8): 647-648.

4. Choi CM, Um SW, Yoo CG, Kim YW, Han SK, Shim YS, Lee CT. Incidence and risk factors of delayed pneumothorax after transthoracic needle biopsy of the lung. Chest 2004;126(5):1516-1521.

5. Traill ZC, Gleeson FV. Delayed pneumothorax after CT-guided percutaneous fine needle aspiration lung biopsy. Thorax 1997;52(6): 581-582.

6. Speich R, van der Bij W. Epidemiology and management of infections after lung transplantation. Clin Infect Dis 2001;33(Suppl 1): S58-S65.

7. Yang DG, Kim KD, Shin DH, Choe KO, Kim SK, Lee WY. Idiopathic bronchiolitis obliterans with organizing pneumonia presenting with spontaneous hydropneumothorax and solitary pulmonary nodule. Respirology 1999;4(3):267-270.

8. Iwanaga $T$, Hirota $T$, Ikeda $T$. Air leak syndrome as one of the manifestations of bronchiolitis obliterans organizing pneumonia. Intern Med 2000;39(2):163-165.

9. Eastridge CE, Hamman JL. Pneumothorax complicated by chronic steroid treatment. Am J Surg 1973;126(6):784-787. 